\title{
PACIENTE CON ESQUIZOFRENIA. DE LA UNIDAD DE HOSPITALIZACIÓN A LA REHABILITACIÓN: ABORDAJE DE ENFERMERÍA
}

\author{
ARTURO ALONSO SEDANOํำ Y MERCEDES PEÑA GRANGER ${ }^{2}$ \\ ${ }^{1}$ Residente de Enfermería de Salud Mental. Hospital General Universitario Gregorio Marañón. Madrid. \\ 2Jefa de la Unidad de Hospitalización Breve. Tutora de residentes de Salud Mental. \\ Hospital General Universitario Gregorio Marañón. Madrid.
}

\section{INTRODUCCIÓN}

La esquizofrenia se encuentra dentro de las diez principales causas de discapacidad en el mundo, y se define como un trastorno psicótico que afecta a la percepción, el pensamiento, el afecto y la conducta de los pacientes, y deteriora su funcionamiento familiar, escolar y social. La rehabilitación cognitiva de los pacientes con esquizofrenia debe incluir el entrenamiento en habilidades sociales, el manejo personal, un programa de ejercicios para incrementar el bienestar general de los pacientes ${ }^{1}$, así como valorar los grados de déficit cognitivos para poder rehabilitarles y reinsertarles en la sociedad con un funcionamiento aceptable ${ }^{2,3}$.

\section{PRESENTACIÓN DEL CASO}

M.T.P. es un paciente de 33 años, natural de Madrid, diagnosticado de esquizofrenia paranoide, con un coeficiente intelectual límite, en seguimiento hospi-

Correspondencia: A. Alonso.

Correo electrónico: alonso-arturo@hotmail.com talario desde 2006. Sin adherencia al centro de salud mental, por lo que es atendido en las consultas del hospital. Ha tenido 48 ingresos en la Unidad de Hospitalización Breve (último en agosto de 2014; máximo periodo sin ingresar: 1 año y 2 meses) por autoagresividad y heteroagresividad verbal en el medio familiar y social, y física hacia objetos, en el contexto de ideación delirante megalomaníaca; ideación de perjuicio hacia los vecinos e identidad fantástica (superhéroes), y fenómenos telepáticos y de lectura de pensamiento, todo ello secundario a consumo de tóxicos, sin motivación para el abandono del consumo. Estos comportamientos le han conducido a tener varios problemas judiciales.

Historia biográfica: Hijo único. Ha convivido con su abuela desde su nacimiento, la cual ha colaborado activamente en su cuidado. Estudios: hasta 2. ${ }^{\circ}$ de ESO. Divorciado, sin hijos. Bien relacionado socialmente, aunque con actividades marginales y delictivas. Madre (damnificada por el aceite de Colza) gran dependiente (en silla de ruedas), con la que presenta una relación patológica (hasta el fallecimiento de esta en 2015) que dificulta la adherencia terapéutica (neurolépticos de liberación prolongada 
por vía intramuscular) y hace imposible la convivencia, por lo que se procede a una incapacitación legal y se le asigna un tutor de la Comunidad Autónoma de Madrid. Padre con diagnóstico de esquizofrenia paranoide.

\section{VALORACIÓN DE ENFERMERÍA AL INGRESO EN LA UNIDAD}

La tabla 1 recoge la valoración de enfermería de acuerdo con el modelo de patrones funcionales de salud de Marjory Gordon.

\section{DIAGNÓSTICOS Y PLANIFICACIÓN}

Se utiliza la taxonomía NANDA (North American Nursing Diagnosis Association) ${ }^{4}$ y la clasificación de diagnósticos por patrones, NOC (Nursing Outcomes Classification) ${ }^{5}$ y NIC (Nursing Intervention Classification) ${ }^{6}$ (tabla 2).

\section{EVALUACIÓN PLAN DE CUIDADOS Y RESULTADOS}

M.T.P. ha estado viviendo en un centro residencial de media-larga estancia donde ha ido alcanzando

Tabla 1. Valoración enfermera por patrones

\begin{tabular}{|l|l|l|l}
\hline Percepción - manejo de la salud & $\begin{array}{l}\text { Alérgico al paracetamol, sin enfermedades somáticas de interés. } \\
\text { Conciencia parcial de enfermedad y los factores que la influyen. } \\
\text { Consumo de alcohol y otros tóxicos. }\end{array}$ \\
\hline Nutricional - metabólico & $\begin{array}{l}\text { Apetito conservado. Sin dieta específica. } \\
\text { Índice de masa corporal superior al rango. }\end{array}$ \\
\hline Eliminación & Dentro de la normalidad. \\
\hline Actividad - ejercicio & $\begin{array}{l}\text { Tabaquismo (20 cigarrillos/día). } \\
\text { Autónomo para las ABVD, pero necesita supervisión. } \\
\text { Dependiente para las AIVD. }\end{array}$ \\
\hline Sueño - descanso & Insomnio ocasional que cede con la medicación habitual. \\
\hline Cognitivo - perceptivo & $\begin{array}{l}\text { Conflicto de decisiones secundaria a conciencia parcial de enfermedad. } \\
\text { Discurso coherente pero con contenido pobre. } \\
\text { Bradipsiquia. }\end{array}$ \\
\hline Autopercepción - autoconcepto & Elevada autoestima con sobrevaloración de sus capacidades. \\
\hline Tolerancia al estrés & $\begin{array}{l}\text { Convive con su madre, con la que mantiene una relación patológica. } \\
\text { Nula red social de apoyo. } \\
\text { No tiene pareja en el momento actual. }\end{array}$ \\
\hline Reconoce conductas de riesgo. \\
\hline Relaciones
\end{tabular}

ABVD: actividades básicas de la vida diaria; AIVD: actividades instrumentales de la vida diaria. 
Tabla 2. Diagnósticos de enfermería y planificación

(00188) Tendencia a adoptar conductas de riesgo para la salud $\mathbf{r} / \mathbf{c}$ apoyo social inadecuado $\mathbf{m} / \mathbf{p}$ fallo en emprender las acciones que prevendrían nuevos problemas de salud

\begin{tabular}{|c|c|c|}
\hline NOC & $\begin{array}{l}\text { (1601) Conducta de cumplimiento. Indicador: } \\
\text { - Acepta el diagnóstico del profesional sanitario } \\
\text { (1300) Aceptación: estado de salud. Indicador: } \\
\text { - Reconocimiento de la realidad de la situación de salud }\end{array}$ & PI: 2, PD: 5, PA: 3 \\
\hline NIC & \multicolumn{2}{|c|}{$\begin{array}{l}\text { (4410) Establecimiento de objetivos comunes. Actividades: } \\
\text { - Ayudar al paciente a identificar objetivos realistas que puedan alcanzarse } \\
\text { - Identificar con el paciente los objetivos de los cuidados } \\
\text { - Explorar con el paciente distintas formas para conseguir los mejores objetivos } \\
\text { - Valorar el nivel de funcionamiento actual del paciente con respecto a cada objetivo } \\
\text { (4360) Modificación de la conducta. Actividades: } \\
\text { - Determinar la motivación al cambio del paciente } \\
\text { - Reforzar las decisiones constructivas respecto a las necesidades sanitarias } \\
\text { - Ofrecer un refuerzo positivo a las decisiones del paciente que han sido tomadas } \\
\text { independientemente } \\
\text { - Ayudar al paciente a identificar los más pequeños éxitos producidos }\end{array}$} \\
\hline
\end{tabular}

(00162) Disposición para mejorar la gestión de la propia salud m/p manifiesta deseo de manejar la enfermedad

(1902) Control del riesgo. Indicadores:

NOC - Modifica el estilo de vida para reducir el riesgo

- Reconoce factores de riesgo

PI: 1, PD: 5, PA: 3

PI: 2, PD: 5, PA: 4

(5230) Aumentar el afrontamiento. Actividades:

- Valorar la comprensión del paciente del proceso de enfermedad

- Utilizar un enfoque sereno, de reafirmación

- Animar al paciente a identificar sus puntos fuertes y sus capacidades

- Proporcionar información objetiva respecto del diagnóstico, tratamiento y pronóstico

- Evaluar la capacidad del paciente para tomar decisiones

(4510) Prevención del consumo de sustancias nocivas. Actividades:

- Determinar las sustancias utilizadas

- Determinar el historial de consumo de drogas/alcohol

- Identificar la existencia de grupos de apoyo en la comunidad para el tratamiento a largo plazo del abuso de sustancias nocivas

- Instruir acerca del consumo de drogas para tratar la sustancia consumida

(00095) Insomnio r/c ansiedad m/p expresión de dificultad para conciliar el sueño

\begin{tabular}{l|l} 
NOC & (0003) Descanso. Indicador: \\
& - Descansado mentalmente
\end{tabular}

(1850) Mejorar el sueño. Actividades:

NIC - Determinar el esquema de sueño/vigilia del paciente

- Ayudar a eliminar las situaciones estresantes antes de irse a la cama 
Tabla 2. Diagnósticos de enfermería y planificación (cont.)

(00126) Conocimientos deficientes r/c limitación cognitiva m/p seguimiento inexacto de las instrucciones

(1803) Conocimiento: proceso de la enfermedad. Indicadores:

\begin{tabular}{l|l|l} 
NOC & - Signos y síntomas de la enfermedad & PI: 1, PD: 5, PA: 2 \\
& - Factores de riesgo & PI: 3, PD: 5, PA: 3 \\
& - Grupos de apoyo disponibles & PI: 1, PD: 5, PA: 3
\end{tabular}

(5602) Enseñanza: proceso de enfermedad. Actividades:

- Evaluar el nivel actual de conocimientos del paciente relacionado con el proceso de enfermedad específico

NIC - Revisar el conocimiento del paciente sobre su estado

- Explorar con el paciente lo que ya ha hecho para controlar los síntomas

- Evitar las promesas tranquilizadoras vacías

- Enseñar al paciente medidas para controlar/minimizar síntomas

(00073) Afrontamiento familiar incapacitante $\mathbf{r} / \mathbf{c}$ relaciones familiares muy ambivalentes $\mathbf{m} / \mathbf{p}$ conductas familiares que son perjudiciales para el bienestar

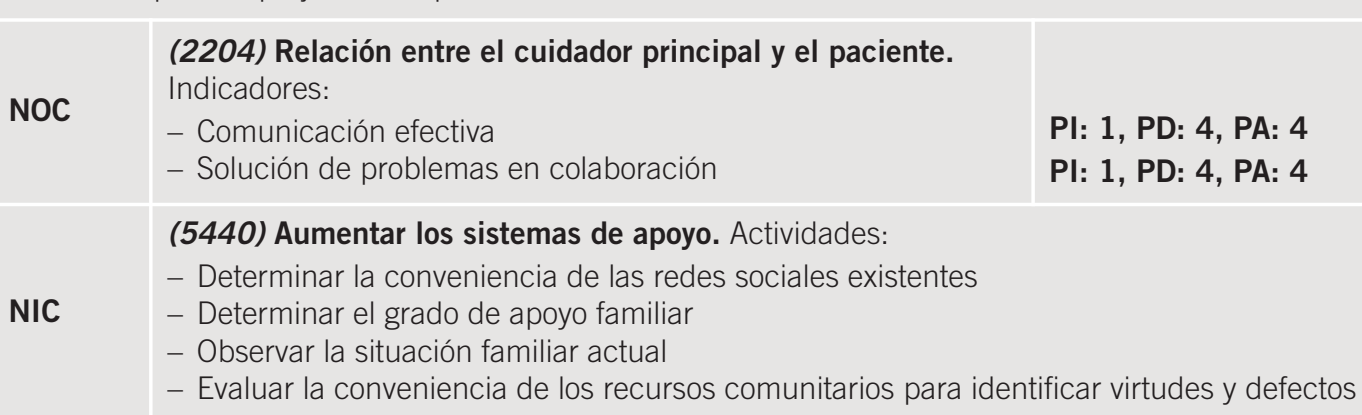

(00114) Síndrome del estrés por traslado r/c deterioro de la salud psicosocial m/p preocupación

\begin{tabular}{l|l|l} 
NOC & $\begin{array}{l}\text { (1305) Modificación psicosocial: cambio de vida. Indicador: } \\
\text { - Establecimientos de objetivos realistas }\end{array}$ & PI: 1, PD: 5, PA: 3 \\
\hline NIC & $\begin{array}{l}\text { (5350) Disminuir el estrés por traslado. Actividades: } \\
\text { - Incluir al individuo en el plan de traslados } \\
\text { - Evaluar el impacto de la interrupción del estilo de vida, pérdida de hogar y adaptación al } \\
\text { nuevo entorno }\end{array}$
\end{tabular}

m/p: manifestado por; PA: puntuación al alta; PD: puntuación deseada; PI: puntuación al ingreso; r/c: relacionado con. Escala Likert: puntuación «5» la mejor posible y «1» la peor posible.

distintas situaciones de autonomía cada vez mayores, reconociendo de manera clara qué factores intervienen en su estabilidad psicopatológica y cuáles influyen en una desestabilización, y sigue con elevada autoestima y con sobrevaloración de sus capa- cidades. Esta evolución ha provocado su traslado a una minirresidencia con muchas posibilidades de pasar, después de valorada su adaptación, a un piso protegido, para seguir trabajando en su autonomía personal. 


\section{BIBLIOGRAFÍA}

1. Ulloa RE, Sauer TB, Apiquian R. Evaluación y tratamiento de la esquizofrenia en niños y adolescentes: una revisión actualizada. Salud Ment. 2011;34(5):42933.

2. Mura G, Petretto DR, Bhat KM, Carta MG. Schizophrenia: from epidemiology to rehabilitation. Clin Pract Epidemiol Ment Health. 2012;8:52.

3. Martín García-Sancho JC, coord. Guía de práctica clínica para el tratamiento de la esquizofrenia en centros de salud mental. Servicio Murciano de la Salud; 2009.
Disponible en: http://www.guiasalud.es/GPC/GPC_ 443_Esquizofrenia_Murcia.pdf

4. Herdman TH, editor. NANDA International. Diagnósticos enfermeros: definiciones y clasificación. 20122014. Barcelona: Elsevier; 2013.

5. Moorhead S, Johnson M, Meridean, ML, Swanson E, editores. Clasificación de resultados de enfermería (NOC). Medición de resultados en salud. 5. ${ }^{a}$ ed. Barcelona: Elsevier; 2013.

6. Bulechek GM, Butcher HK, Dochterman JM, Wagner $\mathrm{CM}$, editores. Clasificación de intervenciones de enfermería (NIC). 6. ${ }^{a}$ ed. Barcelona: Elsevier; 2013. 